\title{
Dense Distributed Processing in a Hindlimb Scratch Motor Network
}

\author{
Robertas Guzulaitis, ${ }^{1,2}$ Aidas Alaburda, ${ }^{2}$ and Jorn Hounsgaard ${ }^{1}$ \\ ${ }^{1}$ University of Copenhagen, Department of Neuroscience and Pharmacology, Copenhagen DK 2200, Denmark, and ${ }^{2}$ Vilnius University, Faculty of Natural \\ Sciences, Department of Neurobiology and Biophysics, Vilnius LT 03101, Lithuania
}

In reduced preparations, hindlimb movements can be generated by a minimal network of neurons in the limb innervating spinal segments. The network of neurons that generates real movements is less well delineated. In an ex vivo carapace-spinal cord preparation from adult turtles (Trachemys scripta elegans), we show that ventral horn interneurons in mid-thoracic spinal segments are functionally integrated in the hindlimb scratch network. First, mid-thoracic interneurons receive intense synaptic input during scratching and behave like neurons in the hindlimb enlargement. Second, some mid-thoracic interneurons activated during scratching project descending axons toward the hindlimb enlargement. Third, elimination of mid-thoracic segments leads to a weakening of scratch rhythmicity. We conclude that densely innervated interneurons in mid-thoracic segments contribute to hindlimb scratching and may be part of a distributed motor network that secures motor coherence.

Key words: dense coding; distributed neural network; scratch reflex; spinal cord; thoracic segments

\section{Introduction}

Processing in sensory neural networks arguably ranges from being local and sparse to distributed and dense (Gire et al., 2013; Petersen and Crochet, 2013). We have previously shown that spinal motoneurons receive intense synaptic input during functional network activity (Alaburda et al., 2005). However, it is not known whether movements are generated by local or distributed processing. Also, the extent of the premotor network involved is unknown. Here we address these questions.

In mammals, the thoracic spinal cord innervates trunk musculature to produce supportive postural adjustments during limb movements. The thoracic segments also mediate descending motor commands to the hindlimbs and propriospinal coordination between forelimbs and hindlimbs. At least in neonates, the passage is not passive but involves and depends on local synaptic circuitry in the thoracic cord (Cowley et al., 2008; Juvin et al., 2012). Recently, recovery of voluntary hindlimb locomotor functions after staggered contralateral hemisections in the thoracic spinal cord in adult rats was shown to depend on extensive rear-

Received March 18, 2014; revised June 27, 2014; accepted July 3, 2014.

Author contributions: R.G. and J.H. designed research; R.G. performed research; R.G., A.A., and J.H. analyzed data; R.G., A.A., and J.H. wrote the paper.

This work was supported by the Lundbeck Foundation, and by Research Council of Lithuania project "Promotion of Student Scientific Activities" (VP1-3.1-ŠMM-01-V-02-003) to R.G., which was funded by the Republic of Lithuania and European Social Fund under the 2007-2013 Human Resources Development Operational Programme's priority 3. We thank Kristian Jensen for assistance with histology, which was performed at the Core Facility of Integrative Microscopy, University of Copenhagen; Tania Rinaldi Barkat for reading the manuscript; and other members of Neuronal Signaling laboratory for commenting on it.

The authors declare no competing financial interests.

Correspondence should be addressed to Dr. Robertas Guzulaitis, University of Copenhagen, Department of Neuroscience and Pharmacology, Panum Institute Blegdamsvej 3B Copenhagen, DK 2200, Denmark. E-mail: r.guzulaitis@gmail.com.

DOI:10.1523/JNEUROSCI.1079-14.2014

Copyright $\odot 2014$ the authors $\quad 0270-6474 / 14 / 3410756-09 \$ 15.00 / 0$ rangement of thoracic circuitry (van den Brand et al., 2012). This use-dependent plasticity somehow enables thoracic neural circuits to fill the gap left by the disrupted direct motor projections to the hindlimb enlargement. These findings suggest that synaptic circuits in the thoracic cord can acquire limb motor function during recovery. However, it is not known whether and how thoracic neurons normally contribite to hindlimb movements. To answer this fundamental question, we have taken advantage of the unique experimental circumstances offered by the adult turtle. First, because turtles lack trunk musculature, their midthoracic segments are devoid of motoneurons and direct motor function (Ruigrok, 1984). For this reason, thoracic segments are thought to serve sensory functions that include communicating sensory input from thoracic dermatomes to limb motor circuits in the hindlimb and forelimb enlargements (Currie and Stein, 1990). Second, because of unusual tolerance to anoxia in adult turtles, neural network activity can be maintained in extensive isolates ex vivo (Kriegstein, 1987; Hounsgaard and Nicholson, 1990). The complete spinal transformation of mechanosensory stimulation into the motor nerve output that generates hindlimb scratching in the intact animal is maintained functional in an isolated carapace-spinal cord preparation (Keifer and Stein, 1983; Alaburda and Hounsgaard, 2003). Here we used the carapace-spinal cord preparation from adult turtles to investigate whether and how mid-thoracic circuitry is engaged in neural network activity underlying rhythmic hindlimb movements.

\section{Materials and Methods}

Ethical approval. The surgical procedures complied with Danish legislation and were approved by the controlling body under the Ministry of Justice.

Ex vivo carapace-spinal cord preparation. Red-eared turtles (Trachemys scripta elegans), $10-15 \mathrm{~cm}$ in carapace length, were obtained from Nasco. Surgical procedures were described previously (Alaburda and Hounsgaard, 
2003). Briefly, turtles ( $n=51)$ of either sex were placed on crushed ice $2 \mathrm{~h}$ before surgery to induce drowsiness and reduce stress and pain by hypothermia. In this way, the head and neck could be protracted using minimal force. Brain functions were terminated immediately upon decapitation by crushing the head. The blood was substituted by perfusion through the heart with a Ringer's solution containing the following (in mM): $120 \mathrm{NaCl}, 5 \mathrm{KCl}, 15 \mathrm{NaHCO}_{3}, 2 \mathrm{MgCl}_{2}, 3 \mathrm{CaCl}_{2}$, and 20 glucose saturated with $98 \% \mathrm{O}_{2}$ and $2 \% \mathrm{CO}_{2}$ to obtain $\mathrm{pH}$ 7.6. Transverse cuts were made at S2 (S for sacral) and D4 (D for dorsal) spinal cord segments. For intracellular recordings from motoneurons, transverse cuts were made at D9-D10 spinal cord segments. The hip flexor nerve (HF) (innervating puboischiofemoralis internus, pars anteroventralis muscle) was exposed and cut.

Stimulation. In the integrated carapace-spinal cord preparation (see Fig. 1A), scratch episodes were initiated by stimulating the carapace and/or skin innervated by afferents of D7/D6 spinal segments (dermatomes from M8.5-M9.5) (Mortin and Stein, 1990). These dermatomes constitute the receptive field for pocket scratching and a transition zone between pocket and rostral scratch (Mortin et al., 1985). In the present project, the fictive scratch activity in motor nerves evoked by mechanical stimulation in these dermatomes is termed pocket scratching. Stimulation was performed with a glass rod mounted on a loudspeaker and controlled by function generator. In some experiments, the scratch reflex was evoked by brushing or pinching pocket skin manually.

Recordings. Intracellular recordings from ventral horn interneurons in segment D4 and motoneurons in segment D9-D10 were performed with a Multiclamp 700B amplifier (Molecular Devices). Sharp glass electrodes from thin-walled borosilicate glass were filled with a mixture of $0.9 \mathrm{M}$ $\mathrm{CH}_{3} \mathrm{CO}_{2} \mathrm{~K}$ and $0.1 \mathrm{M} \mathrm{KCl} ; 2 \%$ biocytin was added to the electrode solution to stain neurons during intracellular recording. The electrode resistance was $40-60 \mathrm{M} \Omega$. Data were sampled at $20 \mathrm{kHz}$ with an analog-to-digital converter (Digidata 1322A, Molecular Devices), displayed by means of Axoscope and Clampex software (Molecular Devices), and stored on a hard disk for later analysis.

Neurons recorded in segments D9-D10 were considered to be motoneurons using the following criteria: neurons were recorded under visual guidance from the motor nucleus in the anterolateral part of ventral horn; their action potential (AP) amplitude was $>80 \mathrm{mV}$ and input resistance $<50 \mathrm{M} \Omega$ (Hounsgaard et al., 1988). Moreover, only neurons with strict in-phase or out-of-phase spike activity with respect to the HF nerve activity during scratching were selected. Because of the indirect identification, we call these neurons putative motoneurons.

The electroneurogram (ENG) of the HF nerve was recorded with a differential amplifier (Iso-DAM8, World Precision Instruments) using a suction electrode. The bandwidth was $100 \mathrm{~Hz}$ to $1 \mathrm{kHz}$.

Biocytin labeling. During experiments, neurons were filled with biocytin ( $2 \%$ in the electrode solution) by passing positive current steps (100$500 \mathrm{pA}, 2 \mathrm{~Hz}, 250 \mathrm{~ms}$ ) for 5-20 min. After filling neurons with biocytin, the D4-D5 spinal segments were removed en bloc from the preparation, kept in PBS with $4 \%$ PFA for $24-48 \mathrm{~h}$ at $4^{\circ} \mathrm{C}$, and subsequently rinsed with and stored in PBS. The fixed tissue was divided longitudinally at the midline separating left and right hemi-cords and glued on an agar block. Then each hemi-cord was sectioned longitudinally into $100-\mu \mathrm{m}$-thick slices using a Leica VT1000S vibratome. The slices were incubated overnight at $4^{\circ} \mathrm{C}$ with cyanine-3-conjugated to streptavidin (1:500, Jackson ImmunoResearch Laboratories) in PBS with $0.3 \%$ Triton X-100 and 5\% donkey serum. After washing in PBS, the slices mounted on glass slides were covered with coverslips on a drop of ProLong Gold antifade reagent (Invitrogen) and cured overnight at room temperature before microscopy.

Micrographs of neurons and their axons were produced using a confocal microscope, Zeiss LSM 700 with diode lasers, on a Zeiss Axiolmager M2 using 10×/0.3 EC Plan-Neofluar and 40×/0.6 EC Plan-Neofluar objectives (Zeiss). The flourophore Cy3 was excited at $555 \mathrm{~nm}$ and detected in the range 560-1000 $\mathrm{nm}$. Images were handled with ZEN 2009 software (Zeiss) in the LSM and 8-bit TIFF format.

Data analysis. The procedure for analyzing periodic spike activity in terms of "preferred phase of AP" (see Fig. 1B) was adapted from others (Berkowitz and Stein, 1994; Stein and Daniels-McQueen, 2002). To find the onset and offset of HF bursts, ENG activity was rectified and smoothed with a sliding $100 \mathrm{~ms}$ window where all data points were weighted equally. The onset and offset were defined as the time points at which the rectified and smoothed ENG crosses 150\% level of baseline ENG activity. Baseline ENG activity was calculated before stimulation. We defined the onset of each HF nerve burst as 0 or 1 and the offset of each HF burst as -0.5 or 0.5 (see Fig. $1 B$ ). The start $(\mathrm{ON})$ and end $(\mathrm{OFF})$ of each burst of APs was calculated with respect to HF activity. At least 3 $\mathrm{ON}$ and OFF phases of bursts of APs during scratch cycles were evaluated for each neuron. Only bursts with $>3$ APs were selected for analysis. The phase of an event (ON or OFF) occurring during HF nerve activity was defined as 0 plus the latency of the event from HF onset divided by twice the duration of the HF nerve burst. The phase of an event (ON or OFF) occurring during HF quiescence was defined as 0.5 plus the latency of the event from HF offset divided by twice the duration of HF nerve quiescence. If an event $(\mathrm{ON})$ preceded $\mathrm{HF}$ onset, then it was defined as -0.5 plus the latency of the event from HF offset divided by twice the duration of HF nerve quiescence. The mid-phase activity was defined as the arithmetic mean between ON and OFF phases. To evaluate the phase shift between scratches (ipsilateral vs contralateral), the difference between mid-phases was calculated.

Conductance of D4 neurons was evaluated from membrane potential $\left(\mathrm{V}_{\mathrm{m}}\right)$ deflections induced by hyperpolarizing $250 \mathrm{~ms}$ current pulses applied at $2 \mathrm{~Hz}$ frequency. To reduce the influence of slow changes in $\mathrm{V}_{\mathrm{m}}$ during the scratch cycle, a reference $V_{m}$ was obtained before and after each hyperpolarizing current pulse. $\mathrm{V}_{\mathrm{m}}$ was averaged during the interval $125-225 \mathrm{~ms}$ of the pulse. To avoid the inherently increased inaccuracy in conductance estimates with increasing conductance, we did not evaluate conductance when $\mathrm{V}_{\mathrm{m}}$ during the injected current step deflected $<10 \%$ from the reference value. This caused us to remove 5 data points in 4 cells ( 3 data points were $>10$ times and 2 data points were $<0$ ) in total. In network reduction experiments, scratch reflex activity was quantified by the number of HF nerve bursts and the frequency of HF bursts. Scratch episodes in preparations containing D3-S2 segments are labeled as "control," and scratch episodes in preparations containing D6-S2 segments are labeled as "D3-D5 cut." In each preparation, scratch episodes were evoked at least 5 times by using the same mechanical stimulus and with a recovery time of $5 \mathrm{~min}$. To evaluate the change in scratch activity after eliminating mid-thoracic segments, the "control" state was compared with the "D3-D5 cut" state.

Data were analyzed statistically using a Student's paired $t$ test (Origin software; Microcal Software). Data are presented as mean \pm SD.

\section{Results}

\section{Mid-thoracic ventral horn interneurons activated during hindlimb scratching}

Mechanical stimuli in dermatomes around the hindlimb evoke a rhythmic motor behavior called scratching (Mortin et al., 1985). This transformation of mechanosensory stimuli into the motor nerve output that generates hindlimb scratching in the intact animal continues to function in an isolated carapace-spinal cord preparation (Fig. 1A) (Keifer and Stein, 1983; Alaburda and Hounsgaard, 2003)

The majority of ventral horn interneurons in the "motoneuronless" mid-thoracic segment D4 receive scratch-related synaptic input during scratch episodes. Although receptive field afferents project to segments D6-D7 (Mortin and Stein, 1990) and hindlimb motor efferents emerge from the hindlimb enlargement D8-S2 (Ruigrok and Crowe, 1984), we found that the synaptic activity increased in 83 of 86 D4 ventral horn interneurons recorded from during scratching. As illustrated in Figure $2 A$, the level of synaptic fluctuations in $\mathrm{V}_{\mathrm{m}}$ was clearly elevated in these cells throughout the scratch episode. Even more striking, slow waves of depolarization were superimposed on the rapid synaptic fluctuations in $60 \%$ of the neurons ( 49 of 83 ). As illustrated in Figure $2 B$, the slow rhythmicity in D4 neurons was correlated with the rhythmic ipsilateral HF motor nerve activity. The suggestion that D4 ventral horn interneurons are integrated in the 

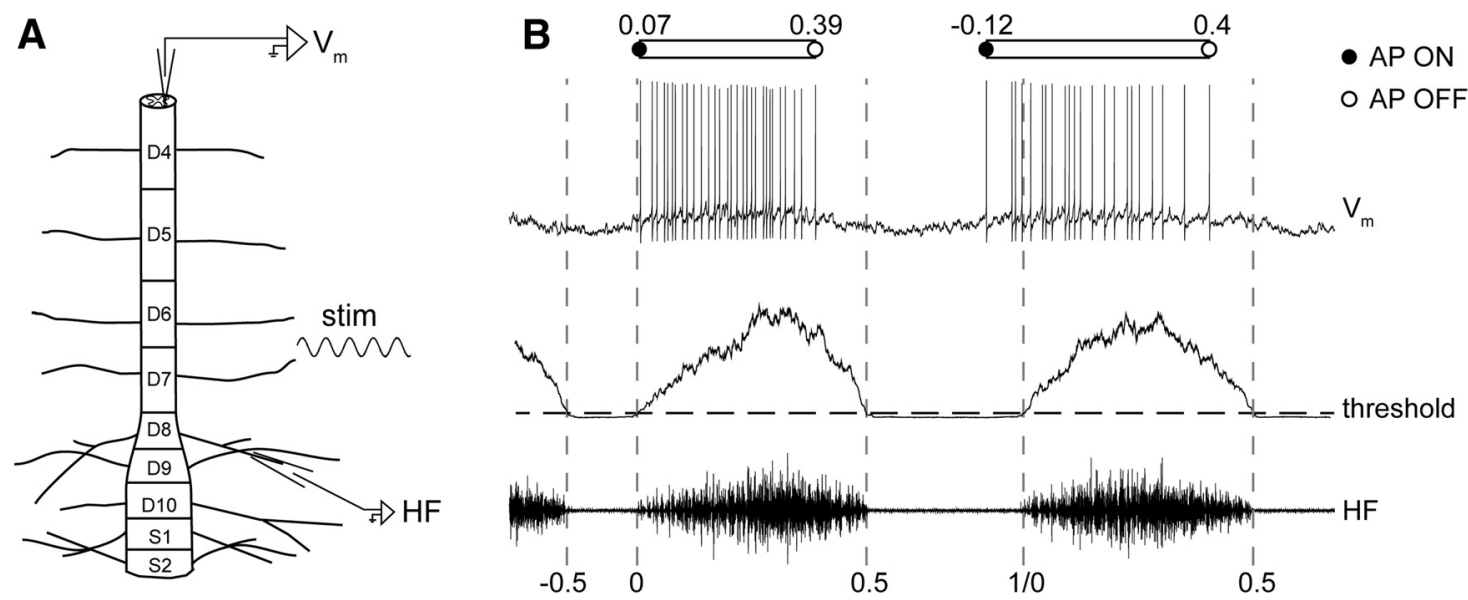

Figure 1. Experimental schematic and scratch phase analysis. Sketch of stimulation and recording arrangement in the integrated carapace-spinal cord preparation from the adult turtle (A). Intracellular recordings in segment D4, scratch rhythm recorded from HF nerve, and stimulation in D6-D7 dermatomes. Evaluation of preferred phase of neuron activity during scratching (B). Onset and end of HF nerve bursts (vertical lines) defined by $150 \%$ of baseline (threshold) of the rectified ENG (middle) of the HF nerve recording (bottom). Intracellular recording of membrane potential $\left(V_{m}\right)$ of neuron in segment D4 (second from top). Top, Filled circles represent onset; open circles represent end of AP burst. Open boxes represent the active phase of neuron during scratch episode.

A
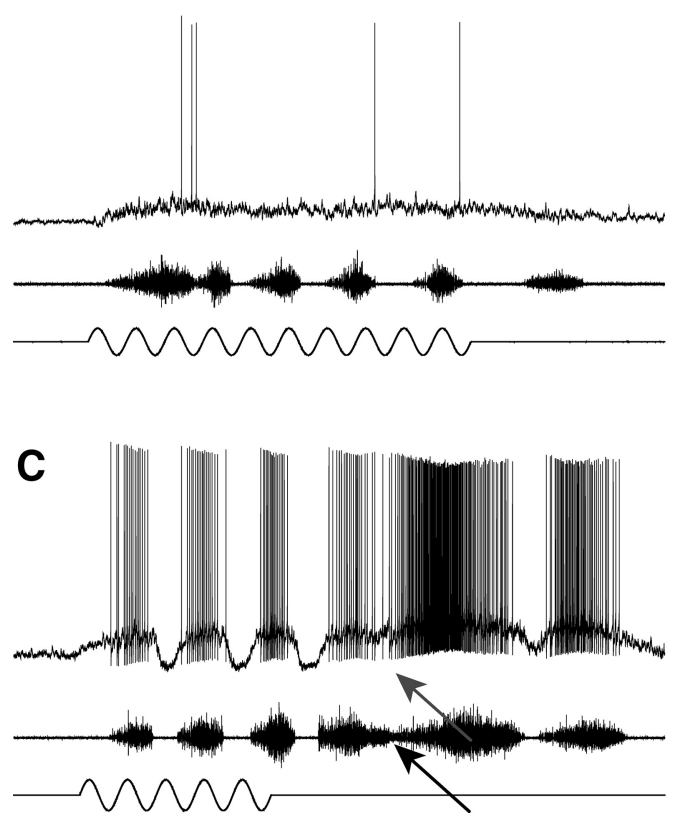

B

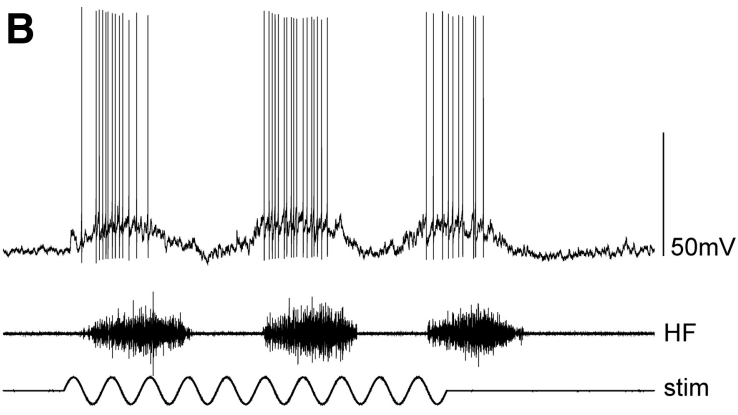

D

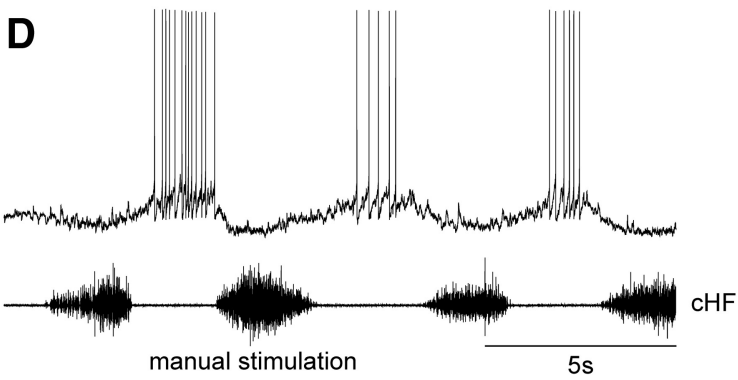

Figure 2. Mid-thoracic ventral horn interneurons activated during hindlimb scratching. Increase in synaptic input during ipsilateral scratching (A). Phasic scratch-related activity (B) and scratch episode with HE deletion ( $C$ ) during ipsilateral scratching. Phasic scratch-related activity during contralateral scratching (D). Top sweep, Intracellular recording from D4 ventral horn interneuron. Middle sweep, HF nerve recording. Bottom sweep, Stimulation signal. C, Black arrow indicates HE deletion observed in HF recording; gray arrow indicates the closely related activity in D4 interneuron (no hyperpolarization) during HE deletion. CHF, Contralateral HF.

scratch motor network was further strengthened by the observation that hip extensor (HE) deletions (Stein, 2008) were clearly reflected in their activity. Figure $2 C$ illustrates one of these rare $(n=3)$ spontaneous deletion episodes observed during the intracellular recordings from the 49 rhythmically active D4 neurons. In the HF motor nerve recording (Fig. $2 C$, middle), an $\mathrm{HE}$ deletion is identified by the fused and prolonged fourth and fifth HF cycles of ENG (black arrow). This deletion is directly reflected in the D4 neuron recorded in parallel by the aborted hyperpolarization and uninterrupted firing between the fused fourth and fifth wave of slow depolarization (gray arrow).

The intimate involvement of D4 ventral horn interneurons in hindlimb scratching was also supported by their response during contralateral scratching. In 47 of 50 D4 neurons tested, the synaptic input increased during contralateral scratching as judged from the increased fluctuations in $\mathrm{V}_{\mathrm{m}}$. Rhythmic depolarizing waves, correlated with the contralateral HF motor nerve activity, were observed in 26 of these neurons (Fig. 2D). To relate activation of D4 neurons during ipsilateral and contralateral scratching, we first analyzed the activity of putative HF and HE motoneurons during ipsilateral and contralaterally induced scratching.

Putative HF and HE motoneuron activity during ipsilateral and contralateral scratching

Hindlimb motor neuron activity during ipsilateral and contralateral fictive scratching has been analyzed in vivo (Stein et al., 1995), 

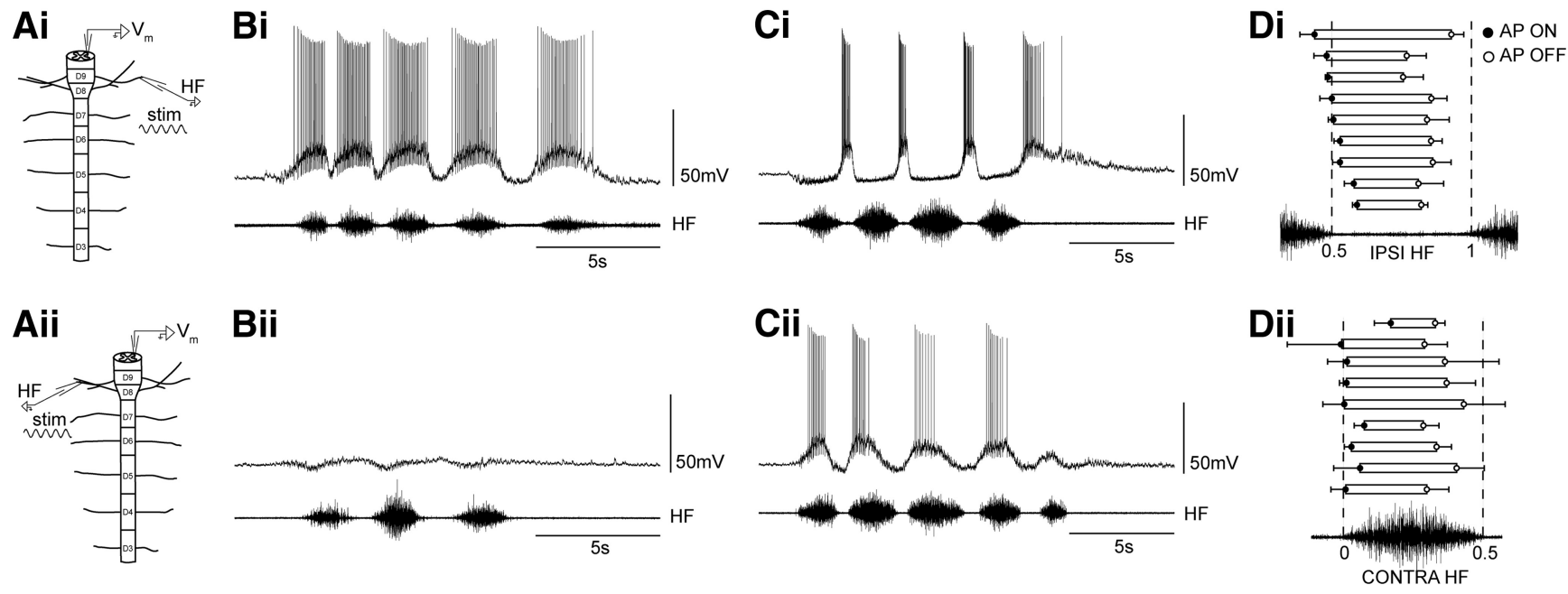

Figure 3. Activity of putative HF and HE motoneurons during ipsilateral and contralateral scratching. Ai, Aii, Experimental arrangement for ipsilateral (Ai) and contralateral (Aii) scratching. Putative HF motoneurons activated during ipsilateral (Bi) and contralateral (Bii) scratching. Putative HE motoneurons activated during ipsilateral (Ci) and contralateral (Cii) scratching. Phase relationship of 9 putative HE motoneurons to HF nerve during ipsilateral (Di) and contralateral (Dii) scratching. $\boldsymbol{B}, \boldsymbol{C}$, Top sweeps, Intracellular recordings of neurons. Bottom sweeps, HF nerve recordings. Filled circles represent onset of D4 neuron firing; open circles represent end of D4 neuron firing. Open boxes represent periods of neuron firing during scratching. Error bars indicate SD of onset and end of neuron firing.

but it is not known to what extent motor pool coordination remains in our ex vivo preparation. In this section, we present intracellular recordings from putative $\mathrm{HF}$ and $\mathrm{HE}$ motoneurons from D9 and D10 segments during ipsilateral and contralateral pocket scratching. Our findings are summarized in Figure 3.

Putative HF motoneurons (i.e., motoneurons activated in phase with the ipsilateral HF nerve activity during ipsilateral scratching) fired vigorously during ipsilateral scratching. Spike trains riding on slow depolarizing waves overlapped the rhythmic nerve activity recorded in the ipsilateral HF nerve (Fig. 3Bi). As illustrated in Figure $3 \mathrm{Bii}$, the slow depolarizing waves were much weaker or absent in all $15 \mathrm{HF}$ neurons tested during contralateral scratching, and spikes were observed in only one of them. In contrast, 12 of 13 putative HE motoneurons (i.e., motoneurons activated out of phase with the ipsilateral HF nerve activity during ipsilateral scratching) fired vigorously during both ipsilateral and contralateral scratching with spike trains riding on slow depolarizing waves (Fig. 3Ci,Cii). The depolarizing waves and the spike activity in putative HE motoneurons alternated out of phase with the ipsilateral HF nerve activity during ipsilateral scratching (Fig. $3 C i, D i$ ) with a mid-phase activity of $0.68 \pm 0.03, n=9$. However, the same putative $\mathrm{HE}$ motoneurons fired in phase with the contralateral HF nerve activity during contralateral scratching (Fig. 3 Cii,Dii) with a mid-phase activity of $0.19 \pm 0.04, n=9$. Our sample putative HE motoneurons were all rhythmically active during ipsilateral and contralateral scratching with a phase shift of $\sim 0.5$ with respect to HF nerve activity $(0.48 \pm 0.04$ in phase shift, $n=9)$. Unilateral stimulation evokes activity in the ispilateral HF motor nerve with minimal activity in contralateral HF motor nerve (Fig. $4 A, B$ ). However, simultaneous stimulation of the left and right receptive fields evokes bilateral scratching with left-right alternating HF nerve activities (Fig. 4C). These findings are in agreement with the out-of-phase interlimb coordination during rostral and pocket scratch forms in vivo (Stein et al., 1995; Field and Stein, 1997). It seems that basic features of the motor pattern during ipsilateral, contralateral, and bilateral scratching are shared by in vivo and ex vivo preparations of the turtle spinal cord. In summary, out-of-phase interlimb coordination during
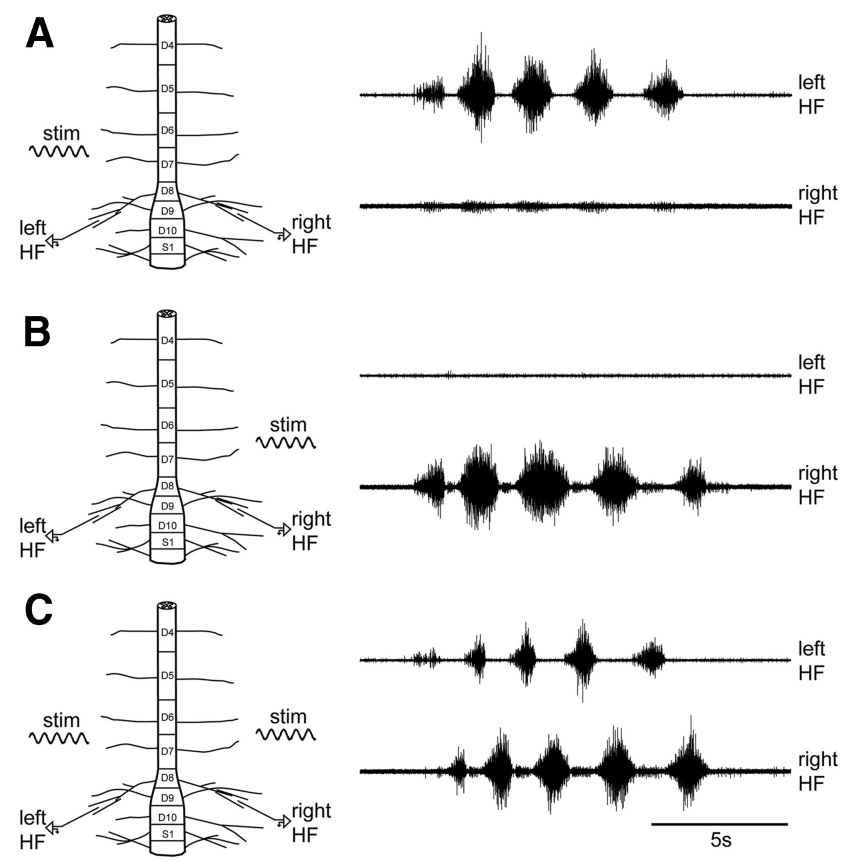

Figure 4. Out-of-phase interlimb coordination during scratching. Unilateral stimulation of scratch receptive fields evokes activity in the ipsilateral HF motor nerve $(\boldsymbol{A}, \boldsymbol{B})$. Simultaneous bilateral stimulation induces alternating activity in the HF nerves $(\boldsymbol{C})$, demonstrating out-ofphase interlimb coordination. A-C, Left, Stimulation and recording. A-C, Right, Activity of HF motor nerves.

pocket scratching is preserved in the integrated carapace-spinal cord preparation.

\section{D4 ventral horn interneuron activity reflects scratch motor patterns}

The phase relationship between AP activity in D4 ventral horn interneurons and HF motor nerve activity during ipsilateral and contralateral scratching was distinctly different from the pattern just described for putative HF and HE motoneurons. The active period of each rhythmic D4 neuron occurred with a cell-specific 

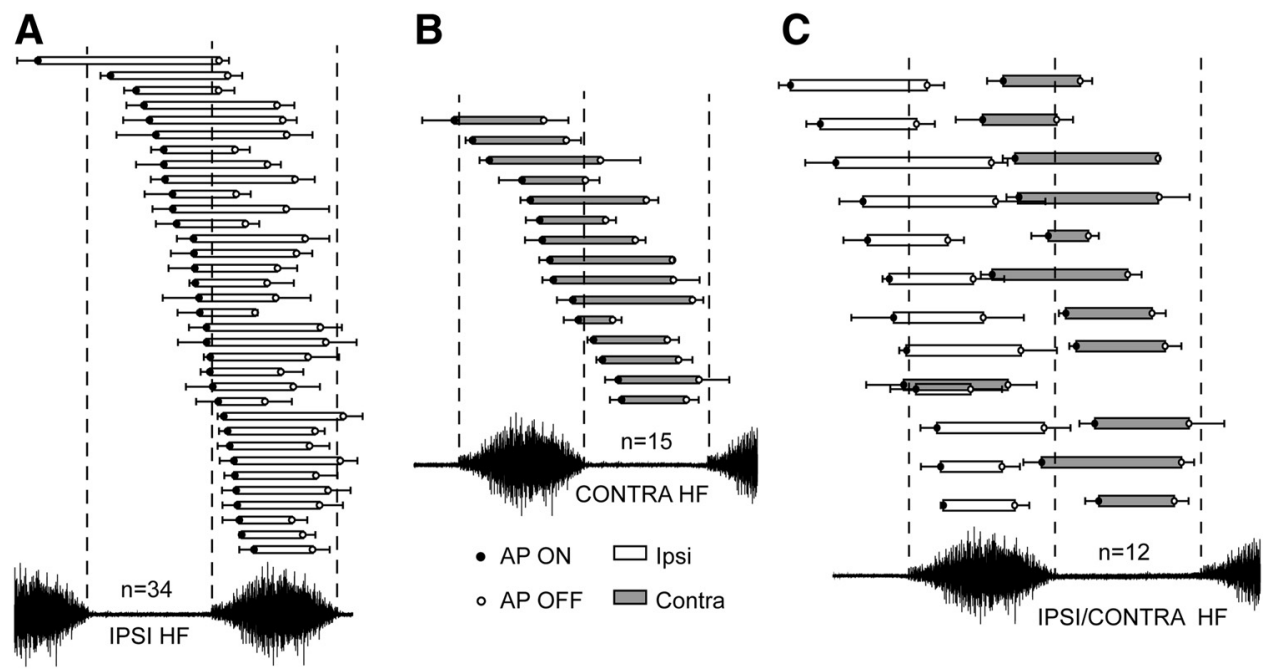

Figure 5. Activity periods of $D 4$ neurons track ipsilateral and contralateral scratching. Phase of activity for $34 \mathrm{D} 4$ neurons during ipsilateral scratching $(\boldsymbol{A})$ and $15 \mathrm{D} 4 \mathrm{neurons}$ during contralateral scratching (B), relative to the normalized HF nerve recording (bottom sweep). Most D4 neurons (11 of 12) are phase-locked to the ipsilateral hip motor cycle during ipsilateral and contralateral scratching $(\boldsymbol{C})$. Filled circles represent onset of $\mathrm{D} 4$ neuron firing; open circles represent end of $\mathrm{D} 4 \mathrm{neuron}$ firing. Open boxes represent periods of neuron firing during ipsilateral scratching; gray boxes represent periods of neuron firing during contralateral scratching. Error bars indicate SD of onset and end of D4 neuron firing. $n=$ number of cells.

phase lag to the HF nerve activity. We performed an activity phase analysis for the rhythmically active D4 interneurons (34 of 49) with clearly separated spike activity during depolarizing waves (see Materials and Methods). Figure $5 \mathrm{~A}$ shows the distribution of active periods for a sample of $34 \mathrm{D} 4$ neurons for ipsilateral scratching. It is apparent that the activity periods normalized to HF nerve cycles (see Materials and Methods) are distributed rather than clustered in a particular phase of the nerve activity. Similar results were obtained for contralateral scratching. In a sample of $15 \mathrm{D} 4$ ventral horn interneurons, the activity periods during contralateral scratching normalized to the contralateral HF nerve cycles also distributed throughout the scratch cycle without obvious clustering (Fig. 5B). Finally, in a sample of 12 D4 interneurons that were rhythmically active during both ipsilateral and contralateral scratching, the active period shifted relative to the normalized HF nerve cycle by 0.5 in 11 cells and was unchanged in 1 (Fig. $5 C$ ) (mid-phase shift was $0.5 \pm 0.15$, $n=12, n=$ number of cells). These findings show that rhythmic activity in the majority of D4 ventral horn interneurons during pocket scratching stays phase-locked to the ipsilateral HF and HE motor pools during ipsilateral and contralateral scratching.

\section{Conductance of D4 neurons during scratching}

During scratching, a substantial increase in conductance attributed to intense synaptic activity has been reported in extensor motoneurons in the cat (Perreault, 2002) and in HF motoneurons and interneurons in the hindlimb enlargement of turtle (Alaburda et al., 2005). In the present study, we noted that the increased synaptic fluctuations in $\mathrm{V}_{\mathrm{m}}$ during scratching were associated with increased conductance as qualitatively judged from the voltage response to small hyperpolarizing current pulses injected through the recording electrode (Fig. $6 A i, B i$ ). This was quantified for 23 phasically active D4 neurons during ipsilateral scratching (Fig. 6Aii) and for 9 phasically active D4 neurons during contralateral scratching (Fig. 6Bii). We found that conductance significantly increases by $47 \pm 34 \%$ during ipsilateral scratching (Student's paired $t$ test, $p=1.5 \times 10^{-6}, n=23, n=$ number of cells) and by $72 \pm 55 \%$ during contralateral scratching (Student's paired $t$ test, $p=4.6 \times 10^{-4}, n=9, n=$ number of cells). We noted that the scratch-induced conductance increase in D4 interneurons was comparable with the increase observed in D9-D10 interneurons (Alaburda et al., 2005). This strongly suggests that synaptic intensity during scratching is of similar magnitude in the two populations of interneurons.

\section{Projections of rhythmic D4 neurons}

Does the rhythmic AP activity in D4 interneurons, seemingly generated by intense ascending synaptic activity during scratching, contribute to the motor network in the hindlimb enlargement or is it an efference copy destined to higher spinal and supraspinal targets? A first crude indication was observed from the axonal projections obtained by biocytin injections in rhythmically active D4 interneurons during scratch network activity. Fourteen D4 interneurons were injected in five preparations. Stained cell bodies and dendrites near the cut rostral end of the spinal cord were observed from 11 of the 14 cells injected (Fig. $7 \mathrm{~A}, \mathrm{Bi}$ ). We do not know whether injection failed in the remaining three cells or they were lost during processing. In five preparations, eight descending axons were observed $>1 \mathrm{~mm}$ from the rostral cut end (illustrated in Fig. 7 Bii,Biii). The identification of descending axons demonstrates that $\mathrm{D} 4$ neurons not only receive input from the scratch network in the hindlimb enlargement but also send their axons back toward neural networks in the hindlimb enlargement. These findings strengthened the possibility that D4 interneurons contribute directly to the generation of hindlimb motor activity.

\section{Contribution of mid-thoracic segments to scratching}

We tested whether elimination of mid-thoracic segments changes scratching as observed in the rhythmic activity recorded in the ipsilateral HF nerve. In four D3-D10 preparations, alternating left and right side scratch episodes were evoked at 5 min intervals by a test stimulus in the hindlimb pocket region innervated by afferents of spinal segments D6-D7 (Fig. 8A). After several control scratch episodes, D3-D5 spinal segments were eliminated at once by transecting the spinal cord at the intersegmental borders. 

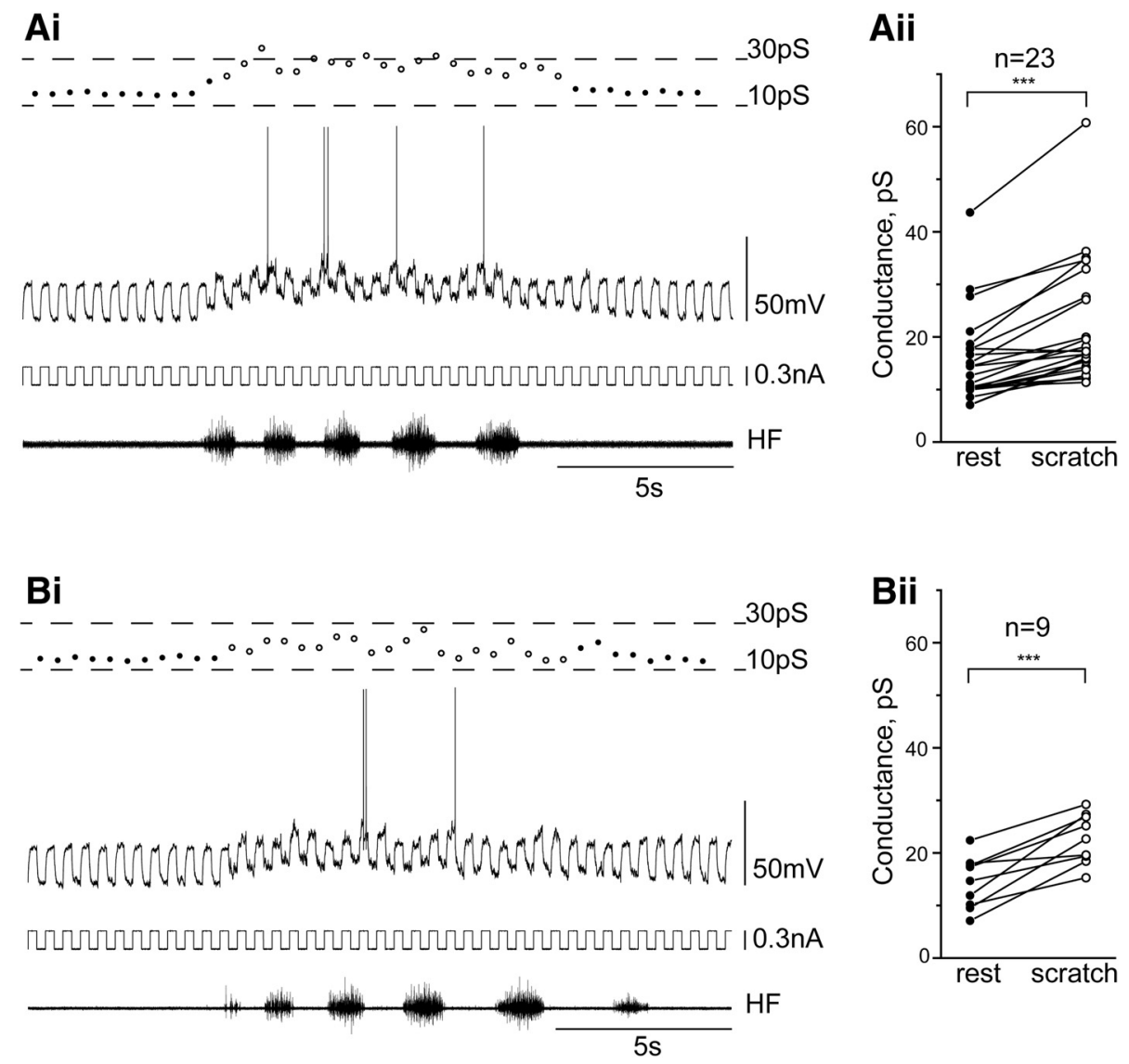

Figure 6. Conductance of D4 neurons during scratching. Conductance increases in rhythmically active D4 neurons during ipsilateral ( $\boldsymbol{A} \boldsymbol{i})$ and contralateral (Bi) scratching. Changes in conductance were quantified for $23 \mathrm{D} 4$ neurons during ipsilateral scratch (Aii) and for $9 \mathrm{D} 4$ neurons (Bii) during contralateral scratch. Conductance increased during scratching $(A, B$, open circles) compared with rest $\left(\boldsymbol{A}, \boldsymbol{B}\right.$, filled circles). $\boldsymbol{A i}, \boldsymbol{B i}$, Recording from the same $\mathrm{D} 4$ neuron. $\boldsymbol{A i}, \boldsymbol{B i}$, Top, Conductance of $\mathrm{D} 4$ neuron, $\mathrm{V}_{\mathrm{m}}$ of $\mathrm{D} 4$ neuron, injected current, $H F$ nerve activity. ${ }^{* * *} p<0.001 . n=$ number of cells.
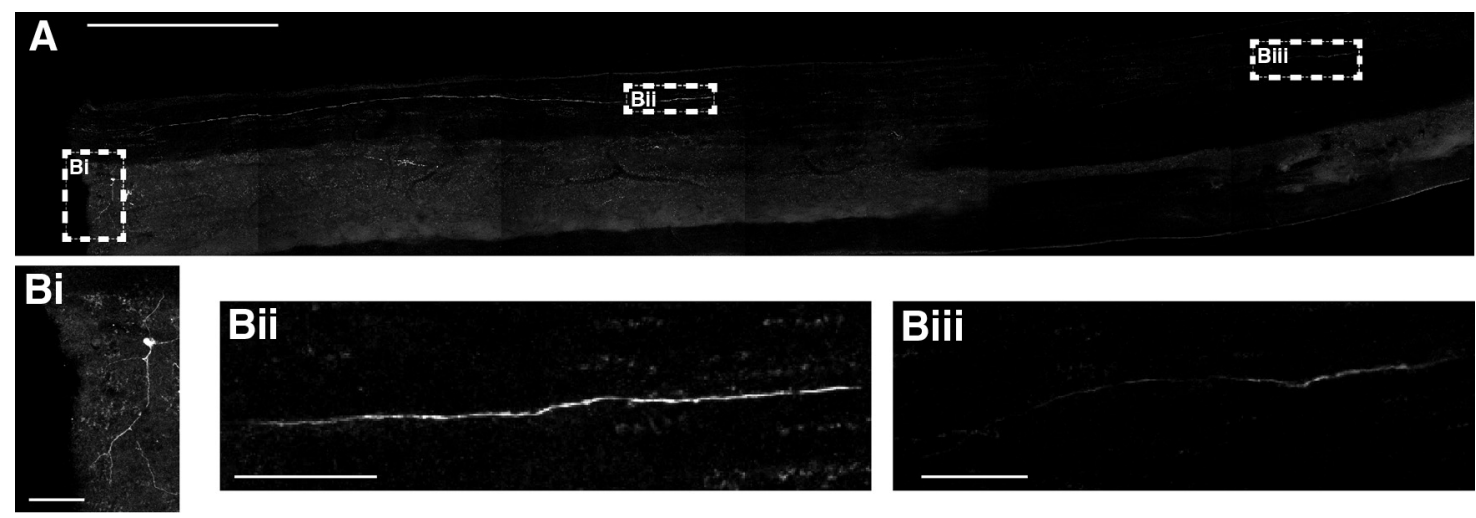

Figure 7. Descending axons from scratch-activated, biocytin-filled D4 neurons. An example of sagittal longitudinal section of D4-D5 spinal segments (A). Cell body (Bi) at the rostral border of slice depicts recording site. Long-range descending axons were recovered from labeled D4 neurons as illustrated in Bii and Biii. Scale bars: $A, 1 \mathrm{~mm} ; \boldsymbol{B}, 0.1 \mathrm{~mm}$.

After a transection, several left and right scratch episodes were elicited again. Scratching was evaluated by measuring the number of HF cycles in each episode and average frequency of HF cycles. The findings are illustrated by recordings from the HF motor nerve in Figure $8 \mathrm{Bi}, \mathrm{Bii}$ and summarized quantitatively in Figure $8 \mathrm{C} i$, Cii for the parameters extracted from all four experiments. Elimination of mid-thoracic segments caused a significant decline in the frequency of scratch cycles (Fig. 8Ci) and in the number of scratch cycles (Fig. 8Cii). In conclusion, elimination of mid-thoracic spinal segments reduces rhythmicity during scratching.

\section{Discussion}

In this study, we found that a majority of mid-thoracic ventral horn interneurons are intensely involved in hindlimb scratch network activity in an ex vivo carapace-spinal cord preparation from the adult turtle. Most of the neurons were rhythmically active during fictive scratching, each with its own characteristic phase relationship to the scratch cycle. The phase of activity of the population of rhythmic D4 neurons covers the entire scratch cycle without distinct flexor and extensor clusters. Axons of a majority of our sample of mid-thoracic neurons involved in hindlimb scratching descend toward the hindlimb enlargement, 
A

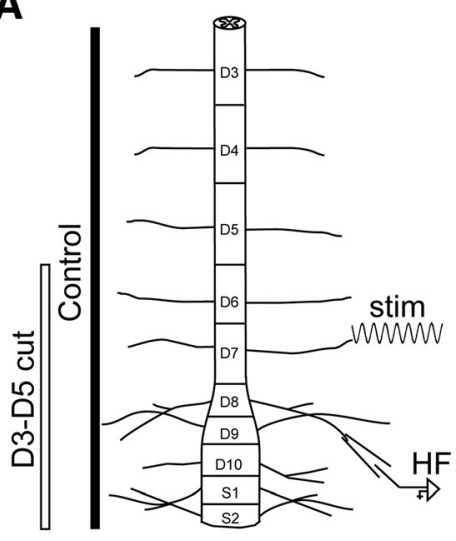

Bi

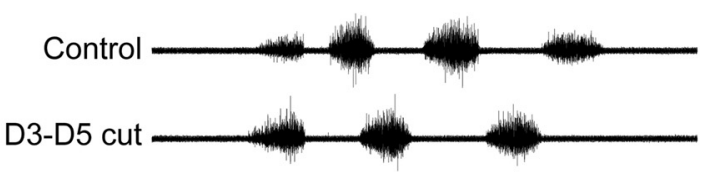

Bii

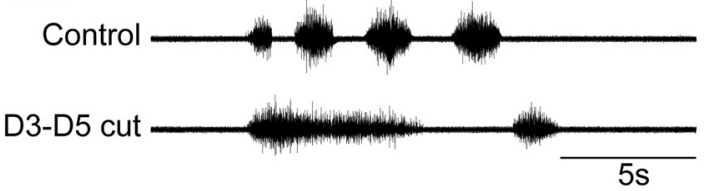

\section{Ci}

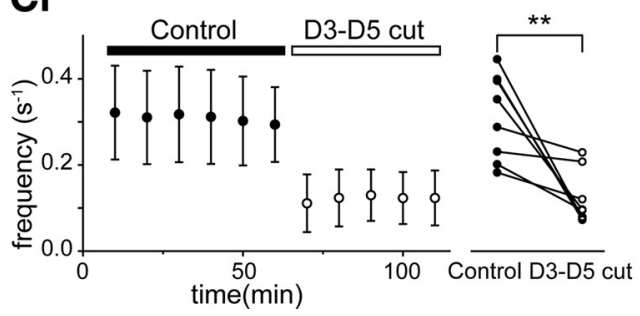

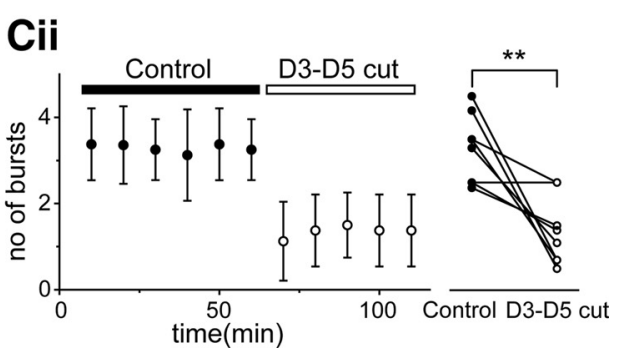

Figure 8. Mid-thoracic segments contribute to hindlimb scratching. Sketch of stimulus and recording arrangement during elimination of thoracic segments $(\boldsymbol{A})$. Scratch episodes monitored from $\mathrm{HF}$ nerve in response to mechanical stimulation in hindlimb pocket region. Examples of reduced scratching in response to elimination of segments D3-D5 in Biand Bii. The same mechanical stimulus evokes fewer and longer HF bursts after removal of thoracic segments (Bi). In some cases, the network responded with a tonic $\mathrm{HF}$ activity to mechanical stimulus after removal of thoracic segments (Bii). Elimination of thoracic segments reduced HF burst frequency per scratch episode ( $\boldsymbol{C}$ ) and number of HF bursts per scratch episode (Cii). HF burst frequency and number of HF bursts throughout experiments in left of $\mathbf{C i}$ and $\mathbf{C i i}$, respectively. Average HF burst frequency and number of HF bursts in control and after removal D3-D5 segments of all experiments in right of Ciand Cii, respectively. "Control" indicates control scratching in D3-S2 preparation; "D3-D5 cut" indicates scratching in D6-S2 preparation. Filled circles represent mean in "Control"; open circles represent mean in "D3-D5 cut" state. Error bars indicate SD of mean. ${ }^{* *} p<0.01$.

and elimination of mid-thoracic segments reduces scratch rhythmicity. These findings show that the hindlimb scratch network in the turtle extends far beyond the hindlimb enlargement and engages in dense distributed processing during functional network activity.

Much effort aimed at defining a core circuit for cyclic hindlimb movements suggests that the minimal rhythmogenic network resides within the hindlimb enlargement (Grillner and Zangger, 1979; Mortin and Stein, 1989; Cazalets et al., 1995). This is supported by increased metabolic activity in neurons of the hindlinmb enlargement during fictive locomotion and scratching (Dai et al., 2005; Mui et al., 2012). It is more difficult, however, to delineate the network that actually contributes to rhythmic limb movements. Obviously, limb movements in intact animals involve posture and tail and interlimb coordination, which makes it difficult to interpret the contribution of network activity beyond the hindlimb enlargement to hindlimb movement per se. However, the rigid trunk and absence of midthoracic motoneurons in the turtle (Ruigrok, 1984) effectively exclude that the scratch-related activity we report for D4 neurons serves local segmental motor function. Furthermore, the reduced scratch activity after eliminating mid-thoracic segments supports extra-hindlimb enlargement involvement in fictive scratch generation. D4 ventral horn interneurons are not just erratically engaged in the scratch activity. They receive intense synaptic activity during scratching as indicated by a similar rhythmicity, increase in synaptic conductance, and fluctuations in $\mathrm{V}_{\mathrm{m}}$ as previously reported for interneurons in the hindlimb enlargement during scratching (Alaburda et al., 2005).
A large fraction of interneurons in the hindlimb enlargement projects ascending axons beyond the hindlimb enlargement (Nissen et al., 2008). There is also ample evidence for direct ascending and descending pathways between the hindlimb enlargement and thoracic segments not only in the turtle (Kusuma and ten Donkelaar, 1980) but also in the cat (Molenaar and Kuypers, 1978; Matsushita et al., 1979) and in the rat (Menétrey et al., 1985; van den Brand et al., 2012). The activation of D4 neurons during hindlimb scratching presented here demonstrates a functional ascending pathway. Moreover, scratch-activated interneurons in mid-thoracic segments project descending axons. Substantial interconnectivity between thoracic and hindlimb-innervating segments provides a substrate for a widely distributed network for hindlimb movements.

Even if ventral horn interneurons in mid-thoracic segments are fully integrated in the scratch circuit, our data offer no direct clues as to their function. It is not known whether they are inhibitory or excitatory, and their postsynaptic targets also remain unknown. However, as a population, they bear striking resemblance to population behavior of interneurons recorded in the hindlimb enlargement during scratching and locomotion. The activity periods of D4 neurons, locked to the hindlimb motor cycle with a cell-specific preferred phase, are shared with interneurons and descending propriospinal axons recorded in the hindlimb enlargement during scratching in the turtle (Berkowitz and Stein, 1994; Stein and Daniels-McQueen, 2002) and the cat (Berkinblit et al., 1978) and during locomotion in the rat (Tresch and Kiehn, 1999) and the cat (Orlovskil and Feldman, 1972; AuY- 
ong et al., 2011a). Like interneurons and descending propriospinal axons in the hindlimb enlargement (Berkowitz and Stein, 1994; Berkowitz, 2001), most D4 interneurons active during ipsilateral and contralateral scratching maintain their preferred phase of activity locked to the ipsilateral motor cycle during ipsilateral and contralateral scratching. Given the similar firing patterns during scratching, it is quite possible that D4 ventral horn interneurons contribute to the axons recorded from the hindlimb enlargement in experiments in vivo (Berkowitz and Stein, 1994; Stein and Daniels-McQueen, 2002).

The wide rostrocaudal distribution and the lack of segmentally specialized firing patterns for rhythmic interneurons in the hindlimb enlargement during scratching and locomotion has been noted (Berkowitz, 2001; Auyong et al., 2011b). The preferred firing phase in mid-thoracic D4 interneurons was evenly distributed over the scratch cycle without clustering. In this sense, they seemingly track rhythmic hindlimb motor network activity as well as interneurons in the hindlimb enlargement (Alaburda et al., 2005; AuYong et al., 2011a). This supports and extends the suggestion that a longitudinal network of interneurons over many segments may constitute a distributed rhythm generator (Rybak et al., 2006; Auyong et al., 2011b). Several observations argue in favor of a distributed rather than local origin of the rhythmic activity in D4 neurons during scratching. First, cutaneous stimulation induced sustained rather than rhythmic firing in descending propriospinal axons in the isolated D4 segment in vivo (Currie and Stein, 1990). Second, elimination of caudal or rostral segments from the scratch network leads to strikingly similar progressively reduced scratch rhythmicity in vivo (Mortin and Stein, 1989) as observed here for elimination of rostral segments ex vivo. These findings are in agreement with the observation that rhythm frequency scales with network size (Mortin and Stein, 1989; Sillar and Roberts, 1993; Wolf and Roberts, 1995).

In conclusion, hindlimb scratching in the turtle engages neurons far beyond the hindlimb enlargement. Our results provide evidence for the idea that rhythm generation in the spinal cord is a distributed global network property (Grillner, 1974; Getting, 1988; Rybak et al., 2006; Auyong et al., 2011b) rather than segmental modules with clockwork-like interactions. A distributed spinal network for rhythmic limb movements offers a substrate for flexible coherence between otherwise disjointed pools of motoneurons and a frame for recovery after spinal lesions. This may offset the high cost of dense distributed processing in large-scale networks (Gire et al., 2013).

\section{References}

Alaburda A, Hounsgaard J (2003) Metabotropic modulation of motoneurons by scratch-like spinal network activity. J Neurosci 23:8625-8629. Medline

Alaburda A, Russo R, MacAulay N, Hounsgaard J (2005) Periodic highconductance states in spinal neurons during scratch-like network activity in adult turtles. J Neurosci 25:6316-6321. CrossRef Medline

AuYong N, Ollivier-Lanvin K, Lemay MA (2011a) Preferred locomotor phase of activity of lumbar interneurons during air-stepping in subchronic spinal cats. J Neurophysiol 105:1011-1022. CrossRef Medline

AuYong N, Ollivier-Lanvin K, Lemay MA (2011b) Population spatiotemporal dynamics of spinal intermediate zone interneurons during airstepping in adult spinal cats. J Neurophysiol 106:1943-1953. CrossRef Medline

Berkinblit MB, Deliagina TG, Feldman AG, Gelfand IM, Orlovsky GN (1978) Generation of scratching: I. Activity of spinal interneurons during scratching. J Neurophysiol 41:1040-1057. Medline

Berkowitz A (2001) Rhythmicity of spinal neurons activated during each form of fictive scratching in spinal turtles. J Neurophysiol 86:1026-1036. Medline

Berkowitz A, Stein PS (1994) Activity of descending propriospinal axons in the turtle hindlimb enlargement during two forms of fictive scratching: phase analyses. J Neurosci 14:5105-5119. Medline

Cazalets JR, Borde M, Clarac F (1995) Localization and organization of the central pattern generator for hindlimb locomotion in newborn rat. J Neurosci 15:4943-4951. Medline

Cowley KC, Zaporozhets E, Schmidt BJ (2008) Propriospinal neurons are sufficient for bulbospinal transmission of the locomotor command signal in the neonatal rat spinal cord. J Physiol 586:1623-1635. CrossRef Medline

Currie SN, Stein PS (1990) Cutaneous stimulation evokes long-lasting excitation of spinal interneurons in the turtle. J Neurophysiol 64:1134-1148. Medline

Dai X, Noga BR, Douglas JR, Jordan LM (2005) Localization of spinal neurons activated during locomotion using the c-fos immunohistochemical method. J Neurophysiol 93:3442-3452. CrossRef Medline

Field EC, Stein PS (1997) Spinal cord coordination of hindlimb movements in the turtle: interlimb temporal relationships during bilateral scratching and swimming. J Neurophysiol 78:1404-1413. Medline

Getting PA (1988) Comparative analysis of invertebrate central pattern generators. In: Neural control of rhythmic movements in vertebrates, pp 101-128. New York: Wiley.

Gire DH, Restrepo D, Sejnowski TJ, Greer C, De Carlos JA, LopezMascaraque L (2013) Temporal processing in the olfactory system: can we see a smell? Neuron 78:416-432. CrossRef Medline

Grillner S (1974) On the generation of locomotion in the spinal dogfish. Exp Brain Res 20:459-470. Medline

Grillner S, Zangger P (1979) On the central generation of locomotion in the low spinal cat. Exp Brain Res 34:241-261. Medline

Hounsgaard J, Nicholson C (1990) The isolated turtle brain and the physiology of neuronal circuits. In: Preparations of vertebrate central nervous system in vitro, pp 155-181. Chichester; NY: Wiley.

Hounsgaard J, Kiehn O, Mintz I (1988) Response properties of motoneurones in a slice preparation of the turtle spinal cord. J Physiol 398:575589. Medline

Juvin L, Le Gal JP, Simmers J, Morin D (2012) Cervicolumbar coordination in mammalian quadrupedal locomotion: role of spinal thoracic circuitry and limb sensory inputs. J Neurosci 32:953-965. CrossRef Medline

Keifer J, Stein PS (1983) In vitro motor program for the rostral scratch reflex generated by the turtle spinal cord. Brain Res 266:148-151. CrossRef Medline

Kriegstein AR (1987) Synaptic responses of cortical pyramidal neurons to light stimulation in the isolated turtle visual system. J Neurosci 7:2488 2492. Medline

Kusuma A, ten Donkelaar HJ (1980) Propriospinal fibers interconnecting the spinal enlargements in some quadrupedal reptiles. J Comp Neurol 193:871-891. CrossRef Medline

Matsushita M, Ikeda M, Hosoya Y (1979) The location of spinal neurons with long descending axons (long descending propriospinal tract neurons) in the cat: a study with the horseradish peroxidase technique. J Comp Neurol 184:63-80. CrossRef Medline

Menétrey D, de Pommery J, Roudier F (1985) Propriospinal fibers reaching the lumbar enlargement in the rat. Neurosci Lett 58:257-261. CrossRef Medline

Molenaar I, Kuypers HG (1978) Cells of origin of propriospinal fibers and of fibers ascending to supraspinal levels: a HRP study in cat and rhesus monkey. Brain Res 152:429-450. CrossRef Medline

Mortin LI, Stein PS (1989) Spinal cord segments containing key elements of the central pattern generators for three forms of scratch reflex in the turtle. J Neurosci 9:2285-2296. Medline

Mortin LI, Stein PS (1990) Cutaneous dermatomes for initiation of three forms of the scratch reflex in the spinal turtle. J Comp Neurol 295:515529. CrossRef Medline

Mortin LI, Keifer J, Stein PS (1985) Three forms of the scratch reflex in the spinal turtle: movement analyses. J Neurophysiol 53:1501-1516. Medline

Mui JW, Willis KL, Hao ZZ, Berkowitz A (2012) Distributions of active spinal cord neurons during swimming and scratching motor patterns. J Comp Physiol A Neuroethol Sens Neural Behav Physiol 198:877-889. CrossRef Medline

Nissen UV, Moldovan M, Hounsgaard J, Glover JC (2008) Organization of projection-specific interneurons in the spinal cord of the red-eared turtle. Brain Behav Evol 72:179-191. CrossRef Medline

Orlovskii GN, Feldman AG (1972) Classification of the neurons of the 
lumbo-sacral region of the spinal cord in accordance with their discharge patterns during provoked locomotion. Neirofiziologiia 4:410-417. Medline

Perreault MC (2002) Motoneurons have different membrane resistance during fictive scratching and weight support. J Neurosci 22:8259-8265. Medline

Petersen CC, Crochet S (2013) Synaptic computation and sensory processing in neocortical layer 2/3. Neuron 78:28-48. CrossRef Medline

Ruigrok TJ, Crowe A (1984) The organization of motoneurons in the turtle lumbar spinal cord. J Comp Neurol 228:24-37. CrossRef Medline

Ruigrok TJH (1984) Organization and morphology of motoneurons and primary afferents in the lumbar spinal cord of the turtle Pseudemys scripta elegans (PhD Thesis): Drukkerij Elinkwijk.

Rybak IA, Shevtsova NA, Lafreniere-Roula M, McCrea DA (2006) Modelling spinal circuitry involved in locomotor pattern generation: insights from deletions during fictive locomotion. J Physiol 577:617-639. CrossRef Medline

Sillar KT, Roberts A (1993) Control of frequency during swimming in Xenopus embryos: a study on interneuronal recruitment in a spinal rhythm generator. J Physiol 472:557-572. Medline
Stein PS (2008) Motor pattern deletions and modular organization of turtle spinal cord. Brain Res Rev 57:118-124. CrossRef Medline

Stein PS, Daniels-McQueen S (2002) Modular organization of turtle spinal interneurons during normal and deletion fictive rostral scratching. J Neurosci 22:6800-6809. Medline

Stein PS, Victor JC, Field EC, Currie SN (1995) Bilateral control of hindlimb scratching in the spinal turtle: contralateral spinal circuitry contributes to the normal ipsilateral motor pattern of fictive rostral scratching. J Neurosci 15:4343-4355. Medline

Tresch MC, Kiehn O (1999) Coding of locomotor phase in populations of neurons in rostral and caudal segments of the neonatal rat lumbar spinal cord. J Neurophysiol 82:3563-3574. Medline

van den Brand R, Heutschi J, Barraud Q, DiGiovanna J, Bartholdi K, Huerlimann M, Friedli L, Vollenweider I, Moraud EM, Duis S, Dominici N, Micera S, Musienko P, Courtine G (2012) Restoring voluntary control of locomotion after paralyzing spinal cord injury. Science 336:1182-1185. CrossRef Medline

Wolf E, Roberts A (1995) The influence of premotor interneuron populations on the frequency of the spinal pattern generator for swimming in Xenopus embryos: a simulation study. Eur J Neurosci 7:671-678. CrossRef Medline 